\title{
Three-potential formalism for the atomic three-body problem
}

\author{
Z. Papp \\ Institute of Nuclear Research of the Hungarian Academy of Sciences, \\ P.O. Box 51, H-4001 Debrecen, Hungary
}

(August 14, 2018)

\begin{abstract}
Based on a three-potential formalism we propose mathematically wellbehaved Faddeev-type integral equations for the atomic three-body problem and descibe their solutions in Coulomb-Sturmian space representation. Although the system contains only long-range Coulomb interactions these equations allow us to reach solution by approximating only some auxiliary short-range type potentials. We outline the method for bound states and demonstrate its power in benchmark calculations. We can report a fast convergence in angular momentum channels.
\end{abstract}

The Faddeev equations are the fundamental equations of the three-body problems. Besides giving a unified formulation, they are superior to the Schrödinger equation in many respects: in incorporating the boundary conditions, in treating the symmetries, in handling the correlations, etc.. Nevertheless, their use in atomic three-body calculations is rather scarce [1 4], and these calculations showed that to reach a reasonable accuracy many channels are needed. So, the belief spread in the community that the Faddeev equations, the fundamental equations of three-body systems, are not well-suited for treating atomic threebody problems and other techniques can do a much better job, at least for bound states. In Refs. 11-4 the Faddeev equations were used in such a form that, the solution could be reached only by some kind of approximation on the long-range Coulomb potential, and thus the convergence were ensured only via the square integrability of the bound-state wave function.

The aim of this paper is to solve the atomic three-body problems by approximating only short-range type interactions. We invoke a newly established "three-potential" formalism and derive such kind of Faddeev-type integral equations which contain only short-range type interactions as source terms. We solve the equations by approximating only the short-range type source terms. We point out that although we are working with finite matrices the wave functions possesses correct three-body Coulomb asymptotics. Finally, as compulsory benchmark cases, we calculate the helium atom, the positronium ion and the muonic hydrogen molecule ion, and will observe a fast convergence with respect to angular momentum channels.

The "three-potential" formalism was designed for solving nuclear three-body problems in the presence of Coulomb interaction. The method was presented first in bound-sate calculations [5] and was extended to below-breakup scattering calculations [6] where the 
notion of the "three-potential" formalism was also introduced. In this solution to the nuclear three-body problem, in the spirit of the two-potential formalism, all long-range interactions, i.e. all Coulomb interactions, are put, à la Noble [7], into the "free" Green's operator. Invoking again the two-potential formalism, the incalculable Faddeev-Noble Green's operator was linked to a simpler channel distorted Green's operator. The "three-potential" formalism, thus consists of repeated applications of the two-potential formalism, and results in integral equations which contain only short-range interactions as source terms.

The Hamiltonian of an atomic three-body system reads

$$
H=H^{0}+v_{\alpha}^{C}+v_{\beta}^{C}+v_{\gamma}^{C},
$$

where $H^{0}$ is the three-body kinetic energy operator and $v_{\alpha}^{C}$ denotes the Coulomb interaction in subsystem $\alpha$. We introduce here the usual configuration-space Jacobi coordinates $\vec{\xi}_{\alpha}$ and $\vec{\eta}_{\alpha} ; \vec{\xi}_{\alpha}$ is the vector connecting the pair $(\beta, \gamma)$ and $\vec{\eta}_{\alpha}$ is the vector connecting the center of mass of the pair $(\beta, \gamma)$ and the particle $\alpha$. Thus $v_{\alpha}^{C}$ only depends on $\xi_{\alpha}$, i.e. $v_{\alpha}^{C}=v_{\alpha}^{C}\left(\xi_{\alpha}\right)$.

The peculiarity of Hamiltonian (1) is that all potentials are of long-range type, and thus the procedure of Refs. [5,6] cannot be applied without any modification. However, the physical role of the Coulomb potential is twofold. Its long-distance part modifies the asymptotic motion, while its short-range part strongly correlates the two-body subsystems. So, we may split the Coulomb potential as a sum of long-range and short-range terms,

$$
v^{C}=v^{(l)}+v^{(s)} .
$$

Short-range and long-range interactions play entirely different roles in integral equations. While short-range interactions contribute to the source terms, long-range interactions should always be put into the Green's operator. Following Faddeev's procedure [8], we split the wave function into three components,

$$
|\Psi\rangle=\left|\psi_{\alpha}\right\rangle+\left|\psi_{\beta}\right\rangle+\left|\psi_{\gamma}\right\rangle
$$

and, for the components, we arrive at the Faddeev-Noble-type equations

$$
\left|\psi_{\alpha}\right\rangle=G_{\alpha}^{(l)}(E)\left[v_{\alpha}^{(s)}\left|\psi_{\beta}\right\rangle+v_{\alpha}^{(s)}\left|\psi_{\gamma}\right\rangle\right]
$$

with a cyclic permutation for $\alpha, \beta, \gamma$. The Green's operator is defined as

$$
\begin{aligned}
G_{\alpha}^{(l)}(z) & =\left(z-H^{0}-v_{\alpha}^{(l)}-v_{\beta}^{(l)}-v_{\gamma}^{(l)}-v_{\alpha}^{(s)}\right)^{-1} \\
& =\left(z-H^{0}-v_{\alpha}^{C}-v_{\beta}^{(l)}-v_{\gamma}^{(l)}\right)^{-1} .
\end{aligned}
$$

In the spirit of the "three-potential" formalism, we relate this Green's operator to the simpler channel distorted Green's operator via the resolvent relation

$$
G_{\alpha}^{(l)}(z)=\widetilde{G}_{\alpha}(z)+\widetilde{G}_{\alpha}(z) U^{\alpha} G_{\alpha}^{(l)}(z),
$$

with $\widetilde{G}_{\alpha}(z)$ and $U^{\alpha}$ defined by

$$
\widetilde{G}_{\alpha}(z)=\left(z-H^{0}-v_{\alpha}^{C}-u_{\alpha}^{(l)}\right)^{-1}
$$


and

$$
U^{\alpha}=v_{\beta}^{(l)}+v_{\gamma}^{(l)}-u_{\alpha}^{(l)}
$$

respectively. Here we have introduced the auxiliary potential $u_{\alpha}^{(l)}$ which is acting in coordinate $\eta_{\alpha}$ and is required to have the asymptotic form

$$
u_{\alpha}^{(l)}\left(\eta_{\alpha}\right) \sim e_{\alpha}\left(e_{\beta}+e_{\gamma}\right) / \eta_{\alpha} \text { as } \eta_{\alpha} \rightarrow \infty .
$$

In fact, it is an effective long-range interaction between the center of mass of the subsystem $\alpha$ (with charge $e_{\beta}+e_{\gamma}$ ) and the third particle (with charge $e_{\alpha}$ ). The importance of $u_{\alpha}^{(l)}$ lies in the fact that it asymptotically compensates the Coulomb-tail of $v_{\beta}^{(l)}+v_{\gamma}^{(l)}$ in Eq. (8), and thus $U^{\alpha}$ decays faster than the Coulomb interaction in the two-body channel $\alpha$. It does not influence the character of the asymptotic motion, so, although it may contain terms which behave like $1 / \eta_{\alpha}^{2}$ as $\eta_{\alpha} \rightarrow \infty$, it can be treated as short-range interaction. So, in Eq. (田) and in Eq. (6), which constitute the basic integral equations of the atomic three-body problem mediated by the "three-potential" formalism, only short-range type interactions are appearing as source terms, thus the equations are mathematically well behaved. They, similarly to the nuclear Coulomb three-body problem [5, 6], can be solved in CoulombSturmian (CS) space representation.

The CS functions, which are the Sturm-Liouville solutions of the hydrogenic problem [9], are defined in configuration-space for some angular momentum state $l$ as

$$
\langle r \mid n l\rangle=\sqrt{\frac{n !}{(n+2 l+1) !}}(2 b r)^{l+1} \mathrm{e}^{-b r} L_{n}^{2 l+1}(2 b r),
$$

where $n=0,1,2, \ldots, L$ represents the Laguerre polynomials and $b$ is a fixed real parameter. With the functions $\langle r \mid \widetilde{n l}\rangle=\langle r \mid n l\rangle / r$ they form a biorthonormal basis.

Since the three-body Hilbert space is a direct sum of two-body Hilbert spaces, the appropriate basis in angular momentum representation may be defined as a the direct product

$$
|n \nu l \lambda\rangle_{\alpha}=\left[|n l\rangle_{\alpha} \otimes|\nu \lambda\rangle_{\alpha}\right]_{L}, \quad(n, \nu=0,1,2, \ldots)
$$

with the CS states of Eq. (10). Here $l$ and $\lambda$ denote the angular momenta of coordinates $\xi$ and $\eta$, respectively, and they are coupled to the total angular momentum $L$. Now the completeness relation takes the form (with angular momentum summation implicitly included)

$$
\mathbf{1}=\lim _{N \rightarrow \infty} \sum_{n, \nu=0}^{N}|\widetilde{n \nu l} \lambda\rangle_{\alpha}{ }_{\alpha}\langle n \nu l \lambda|=\lim _{N \rightarrow \infty} \mathbf{1}_{N}^{\alpha}
$$

where $\left\langle\xi_{\alpha} \eta_{\alpha} \mid \widetilde{n \nu l} \lambda\right\rangle_{\alpha}=1 / \xi_{\alpha} \eta_{\alpha}\left\langle\xi_{\alpha} \eta_{\alpha} \mid n \nu l \lambda\right\rangle_{\alpha}$. It should be noted that in the three-particle Hilbert space we can introduce three equivalent bases belonging to fragmentation $\alpha, \beta$ and $\gamma$.

In equations (4) we make the following approximation:

$$
\left|\psi_{\alpha}\right\rangle=G_{\alpha}^{(l)}(E)\left[\mathbf{1}_{N}^{\alpha} v_{\alpha}^{(s)} \mathbf{1}_{N}^{\beta}\left|\psi_{\beta}\right\rangle+\mathbf{1}_{N}^{\alpha} v_{\alpha}^{(s)} \mathbf{1}_{N}^{\gamma}\left|\psi_{\gamma}\right\rangle\right]
$$


i.e. we approximate the short-range potential $v_{\alpha}^{(s)}$ in the three-body Hilbert space by a separable form

$$
v_{\alpha}^{(s)} \approx \sum_{n, \nu, n^{\prime}, \nu^{\prime}=0}^{N}|\widetilde{n \nu l} \lambda\rangle_{\alpha} \underline{v}_{\alpha \beta}^{(s)}{ }_{\beta}\left\langle n^{\prime} \widetilde{\nu^{\prime} l^{\prime}} \lambda^{\prime}\right|
$$

where $\underline{v}_{\alpha \beta}^{(s)}=\left(1-\delta_{\alpha \beta}\right)_{\alpha}\left\langle n \nu l \lambda\left|v_{\alpha}^{(s)}\right| n^{\prime} \nu^{\prime} l^{\prime} \lambda^{\prime}\right\rangle_{\beta}$. In Eq. (14) the ket and bra states are defined for different fragmentations depending on the environments of the potential operators in the equations.

Multiplied by the CS states ${ }_{\alpha}\langle\widetilde{n \nu l} \lambda|$ from the left, Eqs. (13) turn into a linear system of homogeneous equations for the coefficients of the Faddeev components $\underline{\psi}_{\alpha}={ }_{\alpha}\left\langle\widetilde{n \nu l} \lambda \mid \psi_{\alpha}\right\rangle$ :

$$
\left\{\left[\underline{G}^{(l)}(E)\right]^{-1}-\underline{v}^{(s)}\right\} \underline{\psi}=\underline{0},
$$

with

$$
\underline{G}_{\alpha}^{(l)}={ }_{\alpha}\left\langle\widetilde{n \nu l} \lambda\left|G_{\alpha}^{(l)}\right| n^{\prime} \widetilde{\nu^{\prime} l^{\prime}} \lambda^{\prime}\right\rangle_{\alpha}
$$

Eq. (15) is solvable if and only if

$$
\operatorname{det}\left\{\left[\underline{G}^{(l)}(E)\right]^{-1}-\underline{v}^{(s)}\right\}=0 .
$$

Notice that the matrix elements of the Green's operator, which contains all long-range terms, are needed only between the same partition $\alpha$ whereas the matrix elements of the short range potentials occur only between different partitions $\alpha$ and $\beta$. The latter can be evaluated numerically by making use of the transformation of Jacobi coordinates [10].

The matrix elements (16) can be obtained by solving the Eq. (6), which is, in fact, a two-body multichannel Lippmann-Schwinger equation. If we perform again the separable approximation (14) on potential $U^{\alpha}$, with the help of the formal solution of Eq. (6) we may now express the inverse matrix $\left(\underline{G}_{\alpha}^{(l)}(E)\right)^{-1}$ as

$$
\left(\underline{G}_{\alpha}^{(l)}\right)^{-1}=\left(\underline{\widetilde{G}}_{\alpha}\right)^{-1}-\underline{U}^{\alpha},
$$

where

$$
\widetilde{G}_{\alpha}={ }_{\alpha}\left\langle\widetilde{n \nu l} \lambda\left|\widetilde{G}_{\alpha}\right| n^{\prime} \widetilde{\nu^{\prime} l^{\prime}} \lambda^{\prime}\right\rangle_{\alpha}
$$

and $\underline{U}^{\alpha}={ }_{\alpha}\left\langle n \nu l \lambda\left|U^{\alpha}\right| n^{\prime} \nu^{\prime} l^{\prime} \lambda^{\prime}\right\rangle_{\alpha}$.

While the latter matrix elements may again be evaluated numerically, for the calculation of the matrix elements in Eq. (19) we proceed as follows. Since we can write the threeparticle free Hamiltonian as a sum of two-particle free Hamiltonians, $H^{0}=h_{\xi_{\alpha}}^{0}+h_{\eta_{\alpha}}^{0}$, the channel distorted Green's operator $\widetilde{G}_{\alpha}$ of Eq. (7) appears as a resolvent of the sum of two commuting Hamiltonians $h_{\xi_{\alpha}}^{C}=h_{\xi_{\alpha}}^{0}+v_{\alpha}^{C}$ and $h_{\eta_{\alpha}}^{(l)}=h_{\eta_{\alpha}}^{0}+u_{\alpha}^{(l)}$, which act in different Hilbert spaces. Thus, according to the convolution theorem [11] we can express the three-body Green's operator $\widetilde{G}_{\alpha}$ by an integral of two-body Green's operators

$$
\widetilde{G}_{\alpha}(z)=\left(z-h_{\xi_{\alpha}}^{C}-h_{\eta_{\alpha}}^{(l)}\right)^{-1}=\frac{1}{2 \pi i} \oint_{C} d z^{\prime}\left(z^{\prime}-h_{\xi_{\alpha}}^{C}\right)^{-1}\left(z-z^{\prime}-h_{\eta_{\alpha}}^{(l)}\right)^{-1} .
$$


Here the contour $C$ should go, in counterclockwise direction, around the continuous and discrete spectrum of $h_{\xi_{\alpha}}^{C}$ in such a way that the resolvent of $h_{\eta_{\alpha}}^{(l)}$ is analytic in the domain encircled by $C$ [cf. Fig. [1]. This condition can only be fulfilled if we define the auxiliary potential $u_{\alpha}^{(l)}$ in such a way that the discrete spectrum of $h_{\eta_{\alpha}}^{(l)}$ does not penetrate into $C$. The matrix elements (19) can be cast into the form

$$
\widetilde{G}(E)=\frac{1}{2 \pi i} \oint_{C} d z^{\prime}{ }_{\alpha}\left\langle\widetilde{n l}\left|\left(z^{\prime}-h_{\xi_{\alpha}}^{C}\right)^{-1}\right| \widetilde{n^{\prime} l^{\prime}}\right\rangle_{\alpha}{ }_{\alpha}\left\langle\widetilde{\nu \lambda}\left|\left(E-z^{\prime}-h_{\eta_{\alpha}}^{(l)}\right)^{-1}\right| \widetilde{\nu^{\prime} \lambda^{\prime}}\right\rangle_{\alpha},
$$

where both matrix elements occurring in the integrand are known from the two-particle case [12].

After solving Eq. (15) for the coefficients $\underline{\psi}$ we can construct the Faddeev components. Considering Eqs. (13), (6) and (20) we get:

$$
\left|\psi_{\alpha}\right\rangle=\left[\frac{1}{2 \pi i} \oint_{C} d z^{\prime}\left(z^{\prime}-h_{\xi_{\alpha}}^{C}\right)^{-1}|\widetilde{n l}\rangle_{\alpha}\left(E-z^{\prime}-h_{\eta_{\alpha}}^{(l)}\right)^{-1}|\widetilde{\nu \lambda}\rangle_{\alpha}\right] \underline{C}_{\alpha}
$$

where $\underline{C}_{\alpha}=\left(\underline{\widetilde{G}}_{\alpha}\right)^{-1}\left(\underline{G}_{\alpha}^{(l)}\right)^{-1}\left[\underline{v}_{\alpha}^{(s)} \underline{\psi}_{\beta}+\underline{v}_{\alpha}^{(s)} \underline{\psi}_{\gamma}\right]$, and the functions in the integrand are also known from two-particle case [12]. So, in this formalism, the Faddeev components appears as a linear combination of a convolution integral of Coulomb-like functions. Since in this procedure we have approximated only short-range type interactions $\left|\psi_{\alpha}\right\rangle$ should possess correct three-body Coulomb asymptotics.

To demonstrate the efficiency of the method we present a few benchmark calculations. As cut-off functions we use the exceptionally smooth error functions and define the auxiliary potentials as:

$$
\begin{aligned}
& v_{\alpha}^{(l)}\left(\xi_{\alpha}\right)=\operatorname{erf}\left(\omega \xi_{\alpha}\right) v_{\alpha}^{C}\left(\xi_{\alpha}\right), \\
& v_{\alpha}^{(s)}\left(\xi_{\alpha}\right)=\operatorname{erfc}\left(\omega \xi_{\alpha}\right) v_{\alpha}^{C}\left(\xi_{\alpha}\right)
\end{aligned}
$$

and

$$
u_{\alpha}^{(l)}\left(\eta_{\alpha}\right)=\Lambda \mathrm{e}^{-\kappa \eta_{\alpha}^{2}}+e_{\alpha}\left(e_{\beta}+e_{\gamma}\right) \operatorname{erf}\left(\omega \eta_{\alpha}\right) / \eta_{\alpha},
$$

where $\omega, \Lambda$ and $\kappa$ are parameters. In defining $u_{\alpha}^{(l)}$, in order to prevent the penetration of the bound-states of $h_{\eta_{\alpha}}^{(l)}$ into the contour $C$, we have added a repulsive gaussian term.

We examine the convergence of the three-body energy with increasing $N$, the number of terms in the expansion of the short-range potentials $v_{\alpha}^{(s)}$ and $U^{\alpha}$. In Table [] we present results for the binding energy for helium atom (with infinitely massive core), positronium ion $\left(e^{-} e^{+} e^{-}\right)$and the muonic hydrogen molecule ion $\left(p p \mu^{-}\right)$. For comparison we give the corresponding result of Faddeev calculations of Ref. [2, 13], where channel-by-channel comparison is possibble, and of Ref. [四], where this kind of comparison is not possibble, and also of results of a very accurate variational calculation [14]. In all cases we can observe that, similarly to what we have experienced with nuclear potentials without and with Coulomb interaction [5], convergence up to 6-7 significant digits is comfortably achieved with terms up to $N \sim 19$ applied for $n$ and $\nu$, and the results are in good agreements with well established 
benchmark results. We can see that with an appropriate choice of $\omega$, a rapid convergence is reached in the partial wave expansion. The convergence in angular momentum channels is much faster then in earlier works [2]. To overcome the poor convergence, the solution of the Faddeev equations in total angular momentum representation was proposed [3, [1], which, however, results in three-dimensional equations. In the light of our results, this seems to be superfluous, since the poor convergence is a consequence of the ill-behavior of the applied form of the Faddeev equations.

It should be noted that in the solution of the Coulomb three-body problem the splitting of the Coulomb interaction into short- and long-range terms were already suggested before [15]. However, this splitting were performed in the three-body configuration space and the resulted Faddeev differential equations were used only for scattering state calculations. In our case the splitting is performed in the subsystem (two-body) Hilbert space and can be effective also for bound-state problems.

We have proposed a set of Faddeev and Lippmann-Schwinger equations for atomic threebody systems using the newly established "three-potential" formalism. The Coulomb interactions were split into long-range and short-range terms and the Faddeev procedure was applied only to the short-range potentials. The resulting modified Faddeev equations are mathematically well behaved since the source terms are of short-range type and all the longrange interactions are kept in the Green's operator. This Green's operator was calculated from the channel distorted Green's operator via two-body multichannel Lippmann-Schwinger equations whose kernels are also of short-range type. Because the channel distorted Green's operator appears as a resolvent of the sum of two commuting two-body Hamiltonians, it can be represented as a convolution integral of the corresponding two-body Green's operators. The use of Coulomb-Sturmian functions is essential as it allows an analytic representation of the two-body Coulomb Green's operator on the complex energy plane, which makes straightforward the calculation of the convolution integral and the incorporation of the infinitely many bound states in an attractive Coulomb potential. In this way the atomic three-body problem, which contains only long-range Coulomb interactions, were solved through approximating only some auxiliary short-range potentials. From this follows that the wave function possesses correct asymptotics. The extension of this formalism to below-breakup scattering calculations is analogous to the nuclear Coulomb case [6]. We hope, that these unique advantages of this method allow us to extend its scope to above-breakup calculations.

This work has been supported by OTKA under Contracts No. T17298 and T020409. 


\section{REFERENCES}

[1] Cravo, E., Fonseca, A.C.: Few-Body Systems 5, 117 (1988); Fonseca, A.C., Peña, M.T.: Phys. Rev. A 38, 4967 (1988).

[2] Schellingerhout, N.W., Kok, L.P., Bosveld, G.D.: Phys. Rev. A. 40, 5568 (1989); Schellingerhout, N.W., Kok, L.P.: Nucl. Phys. A. 508 299c (1990).

[3] Kvitsinsky, A.A., Hu, C.-Y., Carbonell, J., Gignoux, C., Merkuriev, S.P.: Few-Body Systems, Suppl. 6, 544 (1992); Hu, C.-Y., Kvitsinsky, A.A., Merkuriev, S.P.: Phys. Rev. A. 45, 2723 (1992);

[4] Kvitsinsky, A.A., and Hu, C.-Y.: Few-Body Systems 12 7, (1992); Kvitsinsky, A.A.: Hyperfine Interactions 82, 59 (1993)

[5] Papp, Z., Plessas, W.: Phys. Rev. C 54, 50 (1996).

[6] Papp, Z.: Phys. Rev. C 55, 1080 (1997).

[7] Noble, J.V.: Phys. Rev. 161, 945 (1967).

[8] See e.g. Glöckle, W.: The Quantum Mechanical Few-Body Problem (Springer, Berlin, Heidelberg, 1983).

[9] Rotenberg, M.: Ann. Phys. (N.Y.) 19, 262 (1962); Adv. Atom. Mol. Phys. 6, 233 (1970).

[10] Balian, R., Brézin, E.: Nuovo Cim. B 2, 403 (1969).

[11] Bianchi, L., Favella, L.: Nuovo Cim. 6, 6873 (1964).

[12] Papp, Z.: J. Phys. A 20, 153 (1987); Phys. Rev. C 38, 2457 (1988); Phys. Rev. A 46, 4437 (1992); Comp. Phys. Comm. 70, 426 (1992); ibid. 70, 435 (1992).

[13] Schellingerhout, N.W., Factorizability in the Numerical Few-Body Problem, PhD Thesis, Groningen, 1996.

[14] Yeremin, A., Florov, A.M., Kutukova, E.B.: Few-Body Systems 4, 111 (1988)

[15] Merkuriev, S.P.: Ann. Phys. (N.Y.) 130, 395 (1980); Acta Physisca Austriaca, Supplementum XXIII, 65 (1981), Faddeev, L.D. and Merkuriev, S.P.: Quantum Scattering Theory for Several Particle Systems, (Kluver, Dordrech, 1993). 


\section{TABLES}

TABLE I. Convergence of the binding energy of the $H e$ atom, the positronium ion $\left(e^{-} e^{+} e^{-}\right)$ and the muonic hydrogen molecule ion $p p \mu^{-}$with increasing basis for the separable expansion taking into account angular momentum states up to $l=0, l=2, l=4, l=6$ and $l=8$. $N$ denotes the maximum number of basis states employed for $n$ and $\nu$. The values for $H e$ atom and positronium are given in atomic units $\left(\hbar=m_{e}=e^{2}=1\right)$ and the values for $p p \mu^{-}$molecule ion are given in modified atomic units $\left(\hbar=m_{\mu}=e^{2}=1\right)$.

\begin{tabular}{|c|c|c|c|c|c|}
\hline \multirow[b]{2}{*}{$N$} & \multicolumn{5}{|c|}{ Angular momentum channels } \\
\hline & $l=0$ & $l=2$ & $l=4$ & $l=6$ & $l=8$ \\
\hline \multicolumn{6}{|c|}{ He atom: $b=5, \omega=1.5, \Lambda=5$ and $\kappa=2$} \\
\hline$\overline{12}$ & 2.892973 & 2.903778 & 2.903733 & 2.903724 & 2.903723 \\
\hline 13 & 2.892976 & 2.903780 & 2.903735 & 2.903726 & 2.903725 \\
\hline 14 & 2.892976 & 2.903781 & 2.903736 & 2.903727 & 2.903725 \\
\hline 15 & 2.892976 & 2.903781 & 2.903736 & 2.903727 & 2.903726 \\
\hline 16 & 2.892977 & 2.903781 & 2.903736 & 2.903727 & 2.903725 \\
\hline 17 & 2.892977 & 2.903781 & 2.903736 & 2.903727 & 2.903725 \\
\hline 18 & 2.892977 & 2.903781 & 2.903736 & 2.903727 & 2.903725 \\
\hline 19 & 2.892977 & 2.903781 & 2.903736 & 2.903727 & 2.903725 \\
\hline \multirow{2}{*}{\multicolumn{2}{|c|}{$\begin{array}{c}\text { Faddeev in Ref. [13] } \\
\text { with L up to } 20:\end{array}$}} & 2.9076746287 & 2.9044691278 & - & 2.9037959292 \\
\hline & & & & & 2.9037224647 \\
\hline \multicolumn{2}{|c|}{ Variational in Ref. [14] } & & & & 2.903724376984 \\
\hline \multicolumn{6}{|c|}{$e^{-} e^{+} e^{-}$ion: $b=0.8, \omega=0.2, \Lambda=5$ and $\kappa=0.3$} \\
\hline$\overline{12}$ & 0.250602 & 0.261753 & 0.261985 & 0.261997 & 0.261998 \\
\hline 13 & 0.250629 & 0.261761 & 0.261992 & 0.262005 & 0.262006 \\
\hline 14 & 0.250621 & 0.261763 & 0.261994 & 0.262006 & 0.262008 \\
\hline 15 & 0.250572 & 0.261758 & 0.261990 & 0.262002 & 0.262003 \\
\hline 16 & 0.250595 & 0.261762 & 0.261993 & 0.262005 & 0.262006 \\
\hline 17 & 0.250562 & 0.261760 & 0.261991 & 0.262003 & 0.262004 \\
\hline 18 & 0.250569 & 0.261760 & 0.261991 & 0.262004 & 0.262005 \\
\hline 19 & 0.250559 & 0.261760 & 0.261991 & 0.262003 & 0.262005 \\
\hline \multicolumn{2}{|c|}{ Faddeev in Ref. [4] } & & & & 0.2620217 \\
\hline \multirow{2}{*}{\multicolumn{2}{|c|}{ Variational in Ref. [14] }} & & & & 0.2620050702314 \\
\hline & & \multicolumn{4}{|c|}{$p p \mu^{-}$ion: $b=2.5, \omega=0.8, \Lambda=20$ and $\kappa=0.8$} \\
\hline$\overline{12}$ & 0.462815 & 0.486698 & 0.492922 & 0.494063 & 0.494309 \\
\hline 13 & 0.458168 & 0.486700 & 0.492921 & 0.494063 & 0.494309 \\
\hline 14 & 0.455523 & 0.486700 & 0.492922 & 0.494063 & 0.494309 \\
\hline 15 & 0.453833 & 0.486695 & 0.492918 & 0.494060 & 0.494306 \\
\hline 16 & 0.452783 & 0.486695 & 0.492918 & 0.494060 & 0.494306 \\
\hline 17 & 0.452065 & 0.486695 & 0.492918 & 0.494060 & 0.494305 \\
\hline 18 & 0.451559 & 0.486695 & 0.492918 & 0.494060 & 0.494305 \\
\hline 19 & 0.451202 & 0.486695 & 0.492918 & 0.494060 & 0.494305 \\
\hline \multicolumn{3}{|c|}{ Faddeev in Ref. [4] } & & & 0.4943867 \\
\hline \multicolumn{3}{|c|}{ Variational in Ref. 14 } & & & 0.4943867 \\
\hline
\end{tabular}




\section{FIGURES}

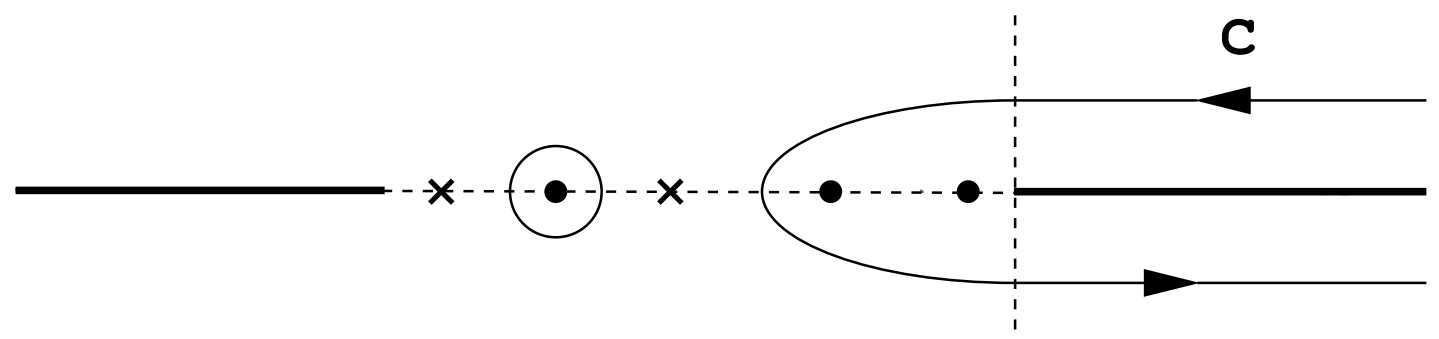

FIG. 1. Contour $C$ for the integral for $\widetilde{G}_{\alpha}(E)$ in case of the three-body bound-state problem with attractive Coulomb interaction. The contour $C$ encircles the continuous and discrete spectrum of $h_{\xi_{\alpha}}^{C}$ and avoids the continuous and discrete spectrum of $h_{\eta_{\alpha}}^{(l)}$. The discrete spectrum of $h_{\xi_{\alpha}}^{C}$ and $h_{\eta_{\alpha}}^{(l)}$ is denoted by dots and crosses, respectively. 